\title{
Neue Veröffentlichungen zum Forschenden Lernen - Eine Sammelrezension
}

\author{
Vanessa Boldt · Alexandra Damm
}

Angenommen: 5. Juli 2021 / Online publiziert: 26. August 2021

(C) Der/die Autor(en) 2021

Forschendes Lernen stellt ein aktuell insbesondere in der Lehrer*innenbildung weit verbreitetes hochschuldidaktisches Konzept dar. Im deutschsprachigen Raum läuft die Debatte über die Potenziale und Grenzen Forschenden Lernens und die Rolle des Konzepts im hochschuldidaktischen Kanon schon seit der Veröffentlichung des zentralen Dokuments der Bundesassistentenkonferenz „Forschendes Lernen Wissenschaftliches Prüfen“ von 1970 (BAK 1970). Der 2011 verabschiedete „Qualitätspakt Lehre“ (QPL) sowie die „Qualitätsoffensive Lehrerbildung“ (QLB) haben dieser Debatte weitere Impulse verliehen. Sowohl die Nachhaltigkeit der frühen konzeptuellen Umsetzungen als auch die aktuellen Entwicklungen verweisen auf die Dynamik und die dem Konzept inhärenten Spannungsfelder u. a. für die Lehre. Verankert in der konstitutiven Idee der Einheit von Forschung und Lehre an Hochschulen wird eine fruchtbare Verbindung zwischen den zwei Polen angestrebt.

Fünf Werke aus den Jahren 2019 und 2020 werden in der vorliegenden Sammelrezension nach alphabetischer Reihung der jeweiligen Herausgebenden vorgestellt. Ein durch die Rezensentinnen verfasster Kriterienkatalog dient dazu, die Bände systematisch miteinander zu vergleichen. Vier Kriterien waren für die Analyse leitend: (1) die disziplinäre Perspektive, unter der Forschendes Lernen thematisiert wird, (2) die Schwerpunkte innerhalb der Publikation, (3) die verwendeten Rahmungen

Die Rezensionen zu Basten et al. (2020), Brinkmann (2019) und Reinmann (2020) wurden von Vanessa-Patricia Boldt, die Rezensionen zu Schiefner-Rohs et al. (2019) und Wulf et al. (2020) von Alexandra Damm angefertigt.

\footnotetext{
Vanessa Boldt $(\bowtie)$

Fakultät für Erziehungswissenschaft, Universität Hamburg, Hamburg, Deutschland

E-Mail: vanessa-patricia.boldt@uni-hamburg.de

Alexandra Damm

Zentrum für Schul- und Bildungsforschung, Martin-Luther-Universität Halle-Wittenberg, Halle, Deutschland

E-Mail: alexandra.damm@zsb.uni-halle.de
} 
bzw. Definitionen von Forschendem Lernen und (4) die angesprochene Zielgruppe. Die fünf Werke werden anhand dieser Kriterien nacheinander dargestellt, ehe die Rezension mit einer Zusammenführung hinsichtlich ihrer Bedeutung für die Lehrer*innenbildung endet.

Basten, Melanie; Mertens, Claudia; Schöning, Anke; Wolf, Eike (Hrsg.): Forschendes Lernen in der Lehrer/innenbildung. Implikationen für Wissenschaft und Praxis. Münster; New York: Waxmann 2020, 279 Seiten, ISBN 978-3-83094154-5, Softcover 29,90€

Der Tagungsband, basierend auf einer im Februar 2019 an der Universität Bielefeld im Rahmen des QLB-Projekts BiProfessional durchgeführten Fachtagung, stellt das Konzept Forschendes Lernen als Gegenstand der lehrer*innenbildungsbezogenen Forschung in das Zentrum verschiedener bildungstheoretischer und fachdidaktischer Perspektiven. Das Werk nähert sich der Definition des Begriffs Forschendes Lernen über die theoretischen Ausprägungen von fachdidaktischen Modellen, deren Umsetzung und der grundlagentheoretischen Diskussion um den Forschungs- und Lernbegriff.

Der einleitende Beitrag (Mertens, Schumacher \& Basten) bietet Systematisierungsversuche und stellt damit dem Band eine metaperspektivische Betrachtung des Begriffes Forschendes Lernen dem gesamten Band voran. Wiederkehrende Motive (u. a. der historische Bezug, ein theoretischer Bezugsrahmen, definitorische Abgrenzungsschwierigkeiten und das Ziel des Erwerbs einer forschenden Grundhaltung) im Diskurs um Forschendes Lernen werden dabei vorgestellt und eingeordnet. Es folgen 28 Einzelbeiträge, die unter den Überschriften (1) „Fachdidaktische LehrLern-Praktiken und Prinzipien“, (2) „Wirksamkeits- und Evaluationsforschung“ sowie (3) „Theoretische Analysen und lehrer/innenbildungsbezogene Implikationen“ versammelt sind. Die begrifflich-theoretischen Grundlagen von unterschiedlichen Konzeptionen Forschenden Lernens werden mit empirischen Untersuchungen der Praxis Forschenden Lernens in universitären Kontexten verknüpft, wobei ein schulpraktischer Bezug beim Forschen (Praxissemester) in den meisten Fällen durch Rückgriff auf den Standort Bielefeld vorliegt. (1) In den vielfältigen fachdidaktischen Beiträgen wird das Konzept des Forschenden Lernen unter anderem im Kontext von Virtueller Realität (Wiesche \& Lipinski) und Lehr-Lern-Laboren (Geisler, Rolka \& da Costa Silva) diskutiert. (2) Die empirischen Erkenntnisse werden vermehrt im Rahmen der Professionalisierungsforschung und anhand der Perspektiven von Lernenden und Lehrenden dargestellt. (3) Ausgehend von theoretischen Modellierungen des hochschuldidaktischen Ansatzes wird eine Typisierung des Forschenden Lernens vorgeschlagen (Katenbrink \& Goldmann). Weitere Beiträge der Überschrift beleuchten die Professionalisierung und Professionalisierungserwartungen in der Lehrer*innenbildung.

Die thematische Breite, die einen Überblick unterschiedlicher Praktiken aus verschiedenen Fachdidaktiken eröffnet ist bemerkenswert und bietet kurze Einblicke in die mannigfaltigen Begriffsbestimmungen und Spezifika Forschenden Lernens in fachspezifischen Lehr-Lernformaten. Die Mehrzahl der Beiträge des Bandes eröffnen dabei ihre spezifische Fokussierung vor einer bestimmten fachdidaktischen Ausrichtung, auch wenn sie nicht der ersten Überschrift zugeordnet sind. Exemplarisch hierfür kann ein Cluster an Beiträgen aus dem Bereich der Sportdidaktik 
angeführt werden, darunter zum Beispiel der Beitrag von Ukley und Bergmann, die unterschiedliche Evaluationsstudien zu den Zusammenhängen von Forschendem Lernen und Professionalisierungsprozessen Sportstudierender zusammenführen und zentrale Erkenntnisse vor einem professionstheoretischen Hintergrund diskutieren. Beiträge mit einem Schwerpunkt auf die Perspektiven von Lehrenden und Studierenden auf das Forschende Lernen ausgehend von empirischen Erkenntnissen geben spannende Einblicke in die Wirksamkeit des Konzepts in den unterschiedlichen Lehr-Settings der Tagungsteilnehmenden. Die Beiträge fokussieren weniger grundsätzlich auf Forschendes Lernen und folgen keinem einheitlichen Aufbau, was eine Kontrastierung verschiedener fachdidaktischer und theoretischer Perspektiven erschwert. Das umfangreiche Bild der Chancen und Herausforderungen der untersuchten Lehr-Lernformate auf theoretischer und empirischer Basis bietet, wie der Titel andeutet, Praktiker*innen und Forscher*innen schnelle Zugänge zu unterschiedlichen Konzeptionen aktueller Hochschulpraxis in der Lehrer*innenbildung.

Brinkmann, Malte (Hrsg.): Forschendes Lernen - Pädagogische Studien zur Konjunktur eines hochschuldidaktischen Konzepts. Phänomenologische Erziehungswissenschaft Band 10, Wiesbaden: Springer Fachmedien, 2019, 140 Seiten, ISBN 978-3-658-28172-4, eBook 29,99€ bzw. Softcover 39,99€

Eingebettet in die Reihe „Phänomenologische Erziehungswissenschaft“ werden im Band Beiträge aus der Lehrer*innenbildung mit dem Ziel einer kritischen Bearbeitung, Rekonstruktion und Reflexion des Konzepts Forschenden Lernens zusammengeführt. Der Sammelband eröffnet keine verschiedenen Rubriken, sondern versammelt sechs Einzelbeiträge mit unterschiedlichen Schwerpunkten, um aktuelle Diskussionen rund um das Forschende Lernen aufzugreifen. Die Wahl des Begriffs „Konjunktur“ im Titel eröffnet die verbindende Schwerpunktsetzung auf einen Diskurs über die gesamte Forschungsentwicklung, mit Blick auf aktuelle positive und kritische Konstruktionen des Konzepts. Das Verhältnis von Forschen, Lehren und Lernen im Kontext der Hochschulen wird mit diskussionsanregender Absicht untersucht, um Herausforderungen in der Legitimation des Konzepts bildungs- und lerntheoretisch sowie didaktisch reflektierend aufzuzeigen und zu bearbeiten. Ein kritischer Entwurf des aktuellen Konzepts des Forschenden Lernens vor der unscharfen grundlagentheoretischen und hochschuldidaktischen Definition wird skizziert und eine Bedeutungs-, Theorie- und Legitimationsproblematik der Lehr- und Lernformate konstatiert.

Der Band wird von einer Einleitung des Herausgebers (Brinkmann) eröffnet. Darin werden die Zielsetzung und Zusammenstellung der einzelnen Beiträge in den Gesamtband eingeordnet. In den Beiträgen werden praxistheoretische, hochschul- und allgemein-didaktische, phänomenologische sowie wissenschaftstheoretische Perspektiven eingenommen. Neben einer historischen Perspektive lassen sich als Schwerpunkte die Zusammenführung von Wissenschaft, Lehrer*innenbildung und Forschung, die Aspekte einer Legitimierung und Umsetzungen in der Praxis sowie Ausblicke auf die Konsequenzen für die Gestaltung identifizieren. Die von den Autor*innen im Band geforderte Zusammenführung von theoretischer und praktischer Umsetzung Forschenden Lernens in der Hochschulbildung soll nach dem Herausgeber als Ausgangspunkt für weitere Anstrengungen genutzt werden. Besonders hervorzuheben sind die bildungstheoretischen und/oder phänomenologischen 
Perspektiven des Bandes auf die Genese des Begriffs und die Modelle des Forschenden Lernens. Exemplarisch hierfür steht der Beitrag von Bellmann, der die Ursprünge des Konzepts von den gesellschaftlichen und politischen Umbrüchen der 1970er-Jahre in der Aktionsforschung bis zu der heutigen Fassung orientiert an Standards und Reformen der Universitäten nachzeichnet und einen Verlust der kritischen Funktion Forschenden Lernens attestiert. Schratz befasst sich mit dem Konzept des „teacher as researcher" anhand empirischer Erkenntnisse, die den Einfluss auf die Orientierung von Lehramtsanwärter*innen fokussieren. Reinmann adaptiert das Modell des didaktischen Dreiecks für die Hochschuldidaktik und bearbeitet dabei die theoretischen und begrifflichen Unschärfen, die im Rahmen der historischen Genese im hochschuldidaktischen Kontext entstanden sind. Eine Verständigung über Beispiele in der Lehre wird von Brinkmann nach einer kritischen Begriffsbetrachtung Forschenden Lernens als Einführung in die wissenschaftliche Forschung herausgearbeitet, während Neuweg die Verbindung von Theorie, Wissen und Wissenschaft mit der Praxis des Lehrens näher beleuchtet und Brenner die Definition einer Wissenschaftsdidaktik schärft.

Das Werk wirft kritische Denkanstöße hinsichtlich der Weiterentwicklung des Konzepts des Forschenden Lernens durch einen historischen Rückblick auf und gibt Anregungen für weitere Bestrebungen einer fundierten Verknüpfung von Lernen, Lehren und Forschen. Die breit entfaltete produktiv-kritische Perspektive kann für theoretische und hochschuldidaktische Forscher*innen sowie Praktiker*innen von Interesse sein.

Reinmann, Gabi; Lübcke, Eileen; Heudorfer, Anna (Hrsg.): Forschendes Lernen in der Studieneingangsphase. Empirische Befunde, Fallbeispiele und individuelle Perspektiven. Wiesbaden: Springer Fachmedien, 2020, 283 Seiten, ISBN 978-3-658-25311-0, eBook 42,99€ bzw. Softcover 54,99€

Der Sammelband, entstanden aus dem Verbundprojekt FideS (Forschungsorientierung in der Studieneingangsphase), folgt der übergeordneten Fragestellung: „Wie geht das: Lernen durch Forschen von Anfang an?" und fokussiert das Konzept des Forschenden Lernens in der Studieneingangsphase an verschiedenen Hochschulen in unterschiedlichen Fachrichtungen. Das Forschende Lernen wird dabei dadurch definiert, dass Studierende zu Beginn ihres Studiums selber forschen. Eine frühe curriculare Verankerung des forschungsnahen Lernens bildet den Schwerpunkt der Beiträge und eröffnet den inhaltlichen Diskurs um Potenziale, Herausforderungen sowie Grenzen aus unterschiedlichen bildungstheoretischen und empirischen Perspektiven der Hochschuldidaktik anhand diverser Fallbeispiele und wird abgeschlossen durch die Perspektive von Expert*innen in Interviews.

Ausführungen zur Einordnung und Ausrichtung des Projekts bilden anstelle einer Einleitung den Beginn des Bandes. Das FideS-Projekt fokussiert neben der genannten Fragestellung den Aufbau von Kooperationsstrukturen unter den Forschenden, die unterschiedliche Akteure einbeziehen, um eine Bündelung von didaktischen Ergebnissen als Synthese aus Erfahrungen, empirischen Befunden und theoretischen Einsichten zur Umsetzung Forschenden Lernens zu generieren. Diesen Ansatz verfolgen die Herausgebenden auch mit dem Sammelband, der neben Erkenntnissen aus dem Projekt Raum für die Vorstellung von Projektpartnern aus unterschiedlichen Disziplinen und Hochschulformen sowie Stimmen von Expert*innen zum 
Forschenden Lernen zusammenführt. Der Sammelband gliedert sich in drei thematische Teilabschnitte mit insgesamt 19 Beiträgen. In Teil A werden zunächst die Ziele, Potenziale und Risiken einer solchen Implementierung in der Studieneingangsphase aufgegriffen (Reinmann, Lübcke \& Heudorfer), ehe die Evaluation Forschenden Lernens diskutiert wird (Favella) und die Rolle von Aspekten wie Neugier (Barnat \& Jänsch), Scheitern (Schiefner-Rohs) und Digitale Medien (Dehne, Knoth \& Lucke) im Kontext des Forschenden Lernen behandelt werden. Die in Teil B vorgestellten Projekte präsentieren in ihrem Vorgehen mögliche Konzeptionen für eine frühe Umsetzung von Forschungsorientierung in der Studieneingangsphase unterschiedlicher Fachrichtungen und Hochschulformen. Die abschließenden Interviews mit Expert*innen in Teil $\mathrm{C}$ geben einen weiteren Einblick in die Modi Forschenden Lernens und vertiefen konkrete Diskussionsthemen durch individuelle Perspektiven, wie es der Titel ankündigt.

Die vorgestellten Projekte und Studiengangkonzepte geben vielfältige Einblicke in Chancen der Umsetzungspraxis Forschenden Lernens am Studienbeginn an deutschen Hochschulen. Der hauptsächliche Fokus auf frühen Forschungserfahrungen gibt dem Werk eine interessante spezifische Schwerpunktsetzung, einhergehend mit einer gleichzeitig daraus entstehenden Limitation einer umfassenden Betrachtung des Konzepts. Dem Band gelingt es, die Diversität des Diskurses zu Forschendem Lernen in der Studieneingangsphase abzubilden und bestehende Praktiken aufzuzeigen, ohne eine thematische Tiefe zu kurz kommen zu lassen.

Schiefner-Rohs, Mandy; Favella, Gianpiero; Herrmann, Anna-Christin (Hrsg.): Forschungsnahes Lehren und Lernen in der Lehrer*innenbildung. Forschungsmethodische Zugänge und Modelle zur Umsetzung. Berlin: Peter Lang, 2019, 250 Seiten, 44,21 €, ISBN 978-3-631-78747-2

Der Band versammelt elf Beiträge zum Thema und startet mit einer Einleitung der Herausgebenden, in welcher sie forschungsnahes Lehren und Lernen in der Lehrer*innenbildung zwischen Wissenschaft, Praxis und Person verorten. Aufgrund der für die Lehrer*innenbildung typischen Verzahnung von Theorie und Praxis hat Forschendes Lernen nach Einschätzung der Herausgebenden bereits eine längere Tradition. Für die Art, wie das Verhältnis von Theorie und Praxis mittels Forschungsorientierung bearbeitet wird, gebe es allerdings verschiedene Varianten. Der Einschätzung der Herausgebenden nach haben bisherige Publikationen zur Thematik entweder vor allem die schulpädagogische Forschung (und damit professionstheoretische Rahmungen und/oder Theorie-Praxis-Implikationen) im Blick oder hochschuldidaktische Ansätze. Der Band nutzt diese Unterscheidung für eine zweigeteilte Strukturierung: In Teil A finden sich Forschungsperspektiven in Form von theoretischen Reflexionen zum Professionalisierungspotential Forschenden Lernens. Teil B richtet sich anhand von Modellen forschungsnahen Lehrens und Lernens vornehmlich an Hochschullehrende innerhalb der Lehrer*innenbildung, Fachwissenschaften und Fachdidaktiken.

So breit angelegt die aufgemachten Forschungsperspektiven sind, so divers sind auch die resultierenden Einschätzungen zu Forschendem Lernen. Während Fichten und Weyland u.a. Schwierigkeiten und Herausforderungen in Bezug auf ihre Bilanz zu vorliegenden Befunden ausmachen und Pollmanns die Möglichkeiten einer Einsozialisation in Wissenschaft kritisch einschätzt, betont Feindt das Reflexions- 
potential Forschenden Lernens und widmen sich Favella und Haunberger den Wirkfaktoren von Forschungsorientierung. Es wäre anzunehmen, dass die Vorstellung unterschiedlicher Modelle forschungsnahen Lehrens und Lernens ebenfalls zu einer sehr diversen Zusammenstellung führen würde, jedoch lassen sich hier zwei Stränge ausmachen: einerseits der Blick auf Unterricht (Jehle, Meßner \& Heiduk) bzw. das Beobachten (de Boer) beim forschungsnahen Lernen und andererseits Professionalisierung durch forschungsnahes Lernen (Katenbrink \& Wischer; Jehle, Meßner \& Heiduk; Bartosch; Gerheim).

Die durch den Band abgebildete Vielschichtigkeit Forschenden Lehrens und Lernens in der Lehrer*innenbildung ist insgesamt als Gewinn für den Diskurs um die Thematik zu betrachten. Jedoch hätte die von den Herausgebenden aufgemachte Verhältnissetzung von Wissenschaft, Praxis und Person ggf. noch stärker nutzbar gemacht werden können. Eine Zuordnung der Beiträge zu einem der drei Pole hätte sich so beispielsweise in einer dreigeteilten Strukturierung des Bandes wiederfinden können. Gleichwohl werden mit der Zweiteilung sehr klar die beiden angesprochenen Zielgruppen des Bandes deutlich: Forschende und Lehrende im Themenfeld forschungsnahen Lehrens und Lernens. Dabei scheint Teil A des Bandes hierbei - entgegen der im Untertitel angekündigten forschungsmethodischen Zugänge jedoch eher forschungstheoretische Perspektiven einzunehmen.

Wulf, Carmen; Haberstroh, Susanne; Petersen, Maren (Hrsg.): Forschendes Lernen. Theorie, Empirie, Praxis. Wiesbaden: Springer VS, 2020, 325 Seiten, 49,99€, ISBN 978-3-658-31489-7

Der Band versammelt - unterteilt in vier Rubriken - insgesamt 26 Beiträge aus verschiedenen Disziplinen zum Thema. Neben theoretisch-didaktischen Begründungen des Konzepts Forschenden Lernens und Beiträgen, die sich der Struktur Forschenden Lernens und damit der curricularen Einbettung widmen (1), finden sich auch empirische Beiträge (2), Beiträge zur praktischen Umsetzung Forschenden Lernens (3) sowie Beiträge unter dem Titel ,Präsentation und Publikation“ (4). Eine gemeinsame zugrunde gelegte Definition Forschenden Lernens ergibt sich über Systematisierungsversuche, die die Herausgebenden im vorgeschalteten Überblick skizzieren. So lassen sich zwei Dimensionen aufspannen, bei denen es einerseits um den inhaltlichen Fokus der Forschungsangebote und andererseits um die Formen der Studierendenbeteiligung geht. Für die im Band versammelten Beiträge gilt laut den Herausgeberinnen eine Orientierung an einem Typus Forschenden Lernens, der die aktive Involviertheit in den gesamten Forschungsprozess betont.

Die Beiträge in der ersten Rubrik verweisen auf An- bzw. Herausforderungen Forschenden Lernens. Hervorzuheben ist hier der Beitrag von Beutel et al., der auch Potenziale in den Blick nimmt (1). Die empirischen Befunde befeuern eine kritische Sicht dabei zum Teil zusätzlich. So etablieren sich beispielsweise laut Wulf et al. keine kognitiven und affektiv-motivationalen Forschungskompetenzen und Spies und Knapp konstatieren einen Widerspruch zwischen Forschendem Lernen und professionellem Selbstverständnis (2). Mit Blick auf die praktische Umsetzung ist insbesondere der Beitrag von Schirmer und Marin interessant, da er anhand von Beispielen und Kriterien aufzeigt, wie digitale Medien in Formaten Forschenden Lernens genutzt werden können (3) - dies scheint disziplinübergreifend und insbesondere in der aktuellen Situation der Pandemie ein nicht zu vernachlässigender 
Fokus zu sein. Die Frage, wie Ergebnisse, die Studierende im Rahmen Forschenden Lernens generieren, sichtbar gemacht werden können, ist der Fokus der letzten Beiträge (4).

Die versammelten Beiträge werden ihrem Ziel - aufzuzeigen, wie vielfältig Forschendes Lernen an deutschen Hochschulen umgesetzt wird - gerecht und bieten zudem Anregungen im Hinblick auf die theoretische, empirische und praktische Auseinandersetzung mit Forschendem Lernen. Dass der Band im Nachgang einer Tagung entstanden ist, an der verschiedene Akteur*innen Forschenden Lernens teilgenommen haben, scheint hier ein besonders fruchtbarer Nährboden gewesen zu sein. Insbesondere im Teil der praktischen Beispiele wird die Breite der Fachdisziplinen, in denen Forschendes Lernen eine Rolle spielt, deutlich. Hier können sich Leser*innen gezielt einzelnen Beiträgen widmen. Damit fokussieren diese Beiträge aber auch weniger grundsätzlich auf Forschendes Lernen. Für den empirischen Teil wäre es wünschenswert gewesen, neben den punktuellen Einblicken auch eine Übersicht über den Forschungsstand zu erhalten. Hilfreich für die Umsetzung Forschenden Lernens ist neben den Praxiseinblicken auch der aus den empirischen Beiträgen resultierende Verweis auf Unterstützungsmöglichkeiten. Besonders positiv hervorzuheben ist zudem ein gewisses Alleinstellungsmerkmal - nicht nur innerhalb des Bandes, sondern auch im Vergleich zu anderen Werken im Kontext Forschenden Lernens: die Sichtbarmachung von studentischen Ergebnissen Forschenden Lernens.

\section{Abschließender Vergleich}

Die Basis für den Vergleich der besprochenen Werke bilden die bereits eingangs genannten Kriterien. Bezüglich der disziplinären Perspektiven, unter denen Forschendes Lernen thematisiert wird, nehmen didaktische Perspektiven einen wesentlichen Stellenwert in den Werken ein. Diese differenzieren sich zwar in allgemein-, fachund hochschuldidaktische Zugänge bzw. bleiben zum Teil unspezifisch bezüglich einer solchen Differenzierung, jedoch ist der fachdidaktische Blickwinkel insgesamt deutlich unterrepräsentiert. Umso stärker ist die Bedeutung des Bandes von Basten et al. (2020), in dem dieser Perspektive dezidiert Platz eingeräumt wird.

Die rezensierten Werke zeichnen sich dabei aber auch allesamt durch eine thematische Breite und vielfältige Zugänge aus. Nichtsdestotrotz lassen sich unterschiedliche Schwerpunkte identifizieren, die von der praktischen Umsetzung des Forschenden Lernens, der Darstellung empirischer Ergebnisse und theoretischer Einordnungen bis hin zu im Rahmen Forschenden Lernens genutzten Methoden reichen. Gleichwohl klären die Werke damit weniger Grundsätzliches in Bezug auf Forschendes Lernen, sondern bieten eher akzentuierte Einblicke in bestimmte disziplin- und perspektivenspezifische Zuschnitte. Damit einhergehende spannende Spezifika (z. B. Forschendes Lernen in der Eingangsphase des Studiums (Reinmann, Lübcke \& Heudorfer 2020), die Publikation von Studierendenergebnissen im Rahmen Forschenden Lernens (Wulf, Haberstroh \& Petersen 2020)) bedeuten aber nicht nur eine Limitation, sondern stellen die Leser*innen gleichzeitig vor die Herausforderung, diese differierenden Anlagen miteinander zu vergleichen. 
Die Vielfältigkeit Forschenden Lernens bedeutet auch, dass es keine allen Werken zugrundeliegende einheitliche Definition Forschenden Lernens gibt. Vielmehr werden verschiedene Systematisierungen vorgenommen, dabei schlagen zum Beispiel Schiefner-Rohs, Favella \& Herrmann (2019) die Verortung Forschenden Lernens zwischen Wissenschaft, Praxis und Person vor. Andere Systematisierungen fokussieren eher Relationen hinsichtlich der Involviertheit in (schulische) Praxis bzw. in wissenschaftliches Handeln (zum Beispiel Wulf, Haberstroh \& Petersen 2020) oder betonen noch stärker, dass ,selber forschen“ eine Grundvoraussetzung sei (zum Beispiel Reinmann, Lübcke \& Heudorfer 2020). Damit steht jedoch letztlich überall die Verhältnissetzung von Eigentätigkeit im Forschen gegenüber anderen oder weiteren Praxisanforderungen im Zentrum.

Während Basten et al. (2020) als Zielgruppe sowohl Praktiker*innen als auch Forscher*innen ansprechen, sind diese bei Brinkmann (2019) nicht ausgenommen, aber werden aufgefordert dezidiert kritisch-produktiv auf Forschendes Lernen zu blicken. Auch Reinmann, Lübcke \& Heudorfer (2020) beziehen sowohl Praktiker*innen und Forscher*innen in ihrer Ansprache mit ein, fokussieren dabei aber die Studieneingangsphase als besonders sensiblen und fruchtbaren Zeitpunkt. Lediglich das Werk von Schiefner-Rohs, Favella \& Herrmann (2019) verortet seine Zielgruppe schon durch seinen Titel explizit in der Lehrer*innenbildung, während bei Wulf, Haberstroh \& Petersen (2020) nur drei Beiträge mit Bezug zum Lehramt vertreten sind. Nichtsdestotrotz bilden beide Werke eine gewisse Breite ab, die sowohl Forschende als auch Lehrende anspricht.

Die unterschiedliche Ausgestaltung, der an die Rezension angelegten Kriterien, eröffnet spannende spezifische Blickwinkel, beinhaltet somit aber auch immer gewisse blinde Flecken. Die Leser*innen sind somit aufgefordert, die jeweilige Relevanz der Werke für die eigene Verortung im Feld Forschenden Lernens einzuschätzen. Die vorliegende Rezension versucht anhand ausgewählter Kriterien einen ersten Zugang dazu ermöglichen.

Funding Open Access funding enabled and organized by Projekt DEAL.

Open Access Dieser Artikel wird unter der Creative Commons Namensnennung 4.0 International Lizenz veröffentlicht, welche die Nutzung, Vervielfältigung, Bearbeitung, Verbreitung und Wiedergabe in jeglichem Medium und Format erlaubt, sofern Sie den/die ursprünglichen Autor(en) und die Quelle ordnungsgemäß nennen, einen Link zur Creative Commons Lizenz beifügen und angeben, ob Änderungen vorgenommen wurden.

Die in diesem Artikel enthaltenen Bilder und sonstiges Drittmaterial unterliegen ebenfalls der genannten Creative Commons Lizenz, sofern sich aus der Abbildungslegende nichts anderes ergibt. Sofern das betreffende Material nicht unter der genannten Creative Commons Lizenz steht und die betreffende Handlung nicht nach gesetzlichen Vorschriften erlaubt ist, ist für die oben aufgeführten Weiterverwendungen des Materials die Einwilligung des jeweiligen Rechteinhabers einzuholen.

Weitere Details zur Lizenz entnehmen Sie bitte der Lizenzinformation auf http://creativecommons.org/ licenses/by/4.0/deed.de. 